\title{
Local Jacquet-Langlands correspondences for simple supercuspidal representations
}

\author{
Naoki Imai and Takahiro Tsushima
}

\begin{abstract}
We give a description of the local Jacquet-Langlands correspondence for simple supercuspidal representations via type theory. As a consequence, we show that the endo-classes for such representations are invariant under the local JacquetLanglands correspondence.
\end{abstract}

\section{Introduction}

Let $K$ be a non-archimedean local field with residue characteristic $p$. Let $A$ be a central simple algebra over $K$. We put $n=[A: K]^{1 / 2}$. The local Jacquet-Langlands correspondence (LJLC) gives a correspondences between irreducible essentially square-integrable representations of $G L_{n}(K)$ and $A^{\times}$. The irreducible supercuspidal representations of $G L_{n}(K)$ is classified in $\mathrm{BK}$ via type theory, which describes supercuspidal representations as compact inductions of representations of some open subgroups that are compact modulo center. More generally, type theory for representations of $A^{\times}$is developed in a series of papers [Se1, Se2], Se3, [SS1], [BSS] and [SS2]. So it is natural to seek a description of the LJLC via type theory.

In the case where $A$ is a division algebra, such descriptions are studied in $\mathrm{Ge}$, [ $\mathrm{He}$, [BH2] and [BH4] if $n$ is a prime number, and in [BH3] if $p$ is odd, $n$ is a power of $p$ and representations are totally ramified. For general $A$, such descriptions are given in [SZ] for level zero discrete series representations, and in [BH5] for essentially tame representations.

In this paper, we give a description of the LJLC via type theory for the simple supercuspidal representations. We define the simple supercuspidal representations of $A^{\times}$ in Definition 1.1 after [RY], which are equivalent to the supercuspidal representations of conductor $n+1$ as a result. Such representations appear in [IT1]. If $A^{\times}=G L_{n}(K)$, simple supercuspidal representations are studied in $\mathrm{AL}$ (cf. $\mathrm{KL}$, $\mathrm{Xu}$ ) and they are called epipelagic representations in [BH6]. We note that the simple supercuspidal representations of $A^{\times}$are essentially tame if $n$ is prime to $p$, but they are not essentially tame if $p$ divides $n$.

As a consequence of the description of the LJLC, we show that the endo-classes are invariant under the LJLC for the simple supercuspidal representations. This verifies

2010 Mathematics Subject Classification. Primary: 11F70; Secondary: 11L05.

Key words: Jacquet-Langlands correspondence, character, Gauss sum, Kloosterman sum 
[BSS, Conjecture 9.5] by Broussous-Sécherre-Stevens for the simple supercuspidal representations. The conjecture is verified in $\mathrm{Ka}$. for totally ramified representations of unit groups of division algebras, based on results in [BH3].

In Section 1, we give a construction and a definition of the simple supercuspidal representations of $A^{\times}$. Further, we show that they are equivalent to the supercuspidal representations of conductor $n+1$. In Section 2, we give formulas for the characters of the simple supercuspidal representations at some elements, which are elliptic quasiregular in the sense of [BH5, 1.1 Remark]. The values of the characters at these elements are written in terms of variants of Gauss sums and generalized Kloosterman sums (cf. Proposition 2.6 and Proposition 2.9). We believe that these formulas are interesting in themselves. In Section 3, we give a description of the LJLC via type theory in Theorem 3.5. We determine the description by checking character relations at some elements. In Section 4, we give another proof of our main theorem using GodementJacquet local constants. This proof is based on a formula in $[\mathrm{Bu}$ and $[\mathrm{BF}]$, which calculates Godement-Jacquet local constants with respect to some kind of Gauss sums. In Section 5, we show the invariance of the endo-classes under the LJLC for the simple supercuspidal representations.

Type theory for irreducible supercuspidal representations of $A^{\times}$naturally appears in the study of geometric realization of the local Langlands correspondence and the LJLC. The results in this paper are used in [IT2] to show that the LJLC is realized in the cohomology of the reductions of a family of affinoids in the Lubin-Tate perfectoid space.

\section{Acknowledgements}

This work was supported by JSPS KAKENHI Grant Numbers 26707003, 15K17506. We thank a referee for suggestions for improvement.

\section{Notation}

For a non-archimedean local field $F$, let $\mathcal{O}_{F}$ denote the ring of integers in $F$. For a field $F$ and a positive integer $l$, let $\mu_{l}(F)$ denote the group of $l$-th roots of the unity inside $F$. For an abelian group $X$, we write $X^{\vee}$ for its character group $\operatorname{Hom}_{\mathbb{Z}}\left(X, \mathbb{C}^{\times}\right)$. For a group $G$, its subgroup $H$, a character $\theta$ of $H$ and $g \in G$, we put $H^{g}=g H g^{-1}$ and define a character $\theta^{g}$ of $H^{g}$ by $\theta^{g}(h)=\theta\left(g^{-1} h g\right)$ for $h \in H^{g}$.

\section{Simple supercuspidal representation}

In this section, we give a construction and a definition of the simple supercuspidal representations of the multiplicative group of a central simple algebra over a nonarchimedean local field. Further, we give a characterization of the simple supercuspidal representations by the conductors.

Let $K$ be a non-archimedean local field with residue field $k$. We set $q=|k|$. Let $\mathfrak{p}_{K}$ be the maximal ideal of $\mathcal{O}_{K}$. Any central simple algebra over $K$ is isomorphic a matrix algebra over a central division algebra over $K$. Let $m$ be a positive integer, and $D$ be a central division algebra over $K$. We put $A=M_{m}(D)$ and $G=A^{\times}$. 
Let $\pi$ be an irreducible smooth representation of $G$. We fix a non-trivial character $\psi \in k^{\vee}$. For $x \in \mathcal{O}_{K}$, let $\bar{x}$ denote the image of $x$ under the reduction map $\mathcal{O}_{K} \rightarrow k$. We take a character $\psi_{K} \in K^{\vee}$ such that

$$
\begin{aligned}
& \psi_{K}(x)=\psi(\bar{x}) \text { for } x \in \mathcal{O}_{K}, \\
& \psi_{K}(x)=1 \text { for } x \in \mathfrak{p}_{K} .
\end{aligned}
$$

Let $\epsilon\left(\pi, s, \psi_{K}\right)$ be the Godement-Jacquet local constant of $\pi$ with respect to $\psi_{K}$. Then there exists an integer $f\left(\pi, \psi_{K}\right)$ such that

$$
\epsilon\left(\pi, s, \psi_{K}\right)=q^{-f\left(\pi, \psi_{K}\right) s} \epsilon\left(\pi, 0, \psi_{K}\right)
$$

by GJ, Theorem 3.3(4)]. We put $r=[D: K]^{1 / 2}$ and $n=m r$. We define the conductor $c(\pi)$ of $\pi$ by

$$
c(\pi)=f\left(\pi, \psi_{K}\right)+n .
$$

Let $\mathcal{A}_{D, m}^{n+1}$ denote the set of the isomorphism classes of the supercuspidal representations of $G$ of conductor $n+1$.

We fix a uniformizer $\varpi$ of $K$. Let $\eta=(\zeta, \chi, c) \in \mu_{q-1}(K) \times\left(k^{\times}\right)^{\vee} \times \mathbb{C}^{\times}$. In the following, we define a smooth representation $\pi_{D, m, \eta}$ of $G$. Let $K_{r}$ be the unramified extension of $K$ of degree $r$. We take an element $\varphi_{D, \zeta} \in D^{\times}$, an embedding $K_{r} \hookrightarrow D$ and an integer $1 \leq s \leq r-1$ which is prime to $r$ such that $\varphi_{D, \zeta}^{r}=\zeta \varpi$ and $\varphi_{D, \zeta} d \varphi_{D, \zeta}^{-1}=d^{q^{s}}$ for $d \in \mu_{q^{r}-1}\left(K_{r}\right)$. We put

$$
\varphi_{\zeta}=\left(\begin{array}{cc}
\mathbf{0} & I_{m-1} \\
\varphi_{D, \zeta} & \mathbf{0}
\end{array}\right) \in A \quad \text { and } \quad L_{\zeta}=K\left(\varphi_{\zeta}\right) \subset A
$$

Since $\varphi_{\zeta}^{n}=\zeta \varpi$, the field $L_{\zeta}$ is a totally ramified extension over $K$ of degree $n$. We have

$$
\operatorname{Nrd}_{A / K}\left(\varphi_{\zeta}\right)=(-1)^{n-1} \zeta \varpi, \quad \operatorname{Trd}_{A / K}\left(\varphi_{\zeta}^{-1}\right)=0
$$

Let $\mathcal{O}_{D}$ denote the maximal order of $D$, and $\mathfrak{p}_{D}$ denote the maximal ideal of $\mathcal{O}_{D}$. For a positive integer $l$, let $k_{l}$ be the extension of $k$ of degree $l$. We identify $\mathcal{O}_{D} / \mathfrak{p}_{D}$ with $k_{r}$. Let $C$ be the subring of $M_{m}\left(k_{r}\right)$ consisting of all upper triangular matrices. Let $\mathfrak{A}$ denote the inverse image of $C$ under the reduction map $M_{m}\left(\mathcal{O}_{D}\right) \rightarrow M_{m}\left(k_{r}\right)$. Then $\mathfrak{A}$ is an order in $A$. Let $\mathfrak{P}_{\mathfrak{A}}$ be the Jacobson radical of $\mathfrak{A}$. Note that $\mathfrak{P}_{\mathfrak{A}}=\varphi_{\zeta} \mathfrak{A}$ and the normalizer of $\mathfrak{A}$ in $G$ equals $L_{\zeta}^{\times} \mathfrak{A}^{\times}$. For a positive integer $i$, we set $U_{\mathfrak{A}}^{i}=1+\mathfrak{P}_{\mathfrak{A}}^{i}$. Let $\theta_{D, m, \eta}: L_{\zeta}^{\times} U_{\mathfrak{A}}^{1} \rightarrow \mathbb{C}^{\times}$be the character defined by

$$
\begin{aligned}
\theta_{D, m, \eta}(x) & =\chi(\bar{x}) \quad \text { for } x \in \mu_{q-1}(K), \\
\theta_{D, m, \eta}(x) & =\left(\psi_{K} \circ \operatorname{Trd}_{A / K}\right)\left(\varphi_{\zeta}^{-1}(x-1)\right) \quad \text { for } x \in U_{\mathfrak{A}}^{1}, \\
\theta_{D, m, \eta}\left(\varphi_{\zeta}\right) & =(-1)^{m-1} c .
\end{aligned}
$$

We put $\pi_{D, m, \eta}=\mathrm{c}-\operatorname{Ind}_{L_{\zeta}^{\times} U_{\mathfrak{A}}^{1}}^{G} \theta_{D, m, \eta}$.

Definition 1.1. We say that an irreducible supercuspidal representation $\pi$ of $G$ is simple supercuspidal if $\pi \simeq \pi_{D, m, \eta}$ for some $\eta \in \mu_{q-1}(K) \times\left(k^{\times}\right)^{\vee} \times \mathbb{C}^{\times}$.

Lemma 1.2. The representation $\pi_{D, m, \eta}$ is a supercuspidal representation of conductor $n+1$. 
Proof. We define a chain lattice $\Lambda=\left\{\Lambda_{i}\right\}_{i \in \mathbb{Z}}$ in $D^{\oplus m}$ by

$$
\Lambda_{m j+l}=\left(\mathfrak{p}_{D}^{j}\right)^{\oplus m-l} \oplus\left(\mathfrak{p}_{D}^{j+1}\right)^{\oplus l}
$$

for $j \in \mathbb{Z}$ and $0 \leq l \leq m-1$. Then we have

$$
\mathfrak{A}=\left\{g \in A \mid g \Lambda_{i} \subset \Lambda_{i} \text { for all } i \in \mathbb{Z}\right\}
$$

and $\varphi_{\zeta} \Lambda_{i} \subset \Lambda_{i+1}$ for $i \in \mathbb{Z}$. Hence, $\mathfrak{A}$ is a hereditary order in $A$ (cf. [Se1, Définition 1.3]). We consider a stratum $\left[\mathfrak{A}, 1,0, \varphi_{\zeta}^{-1}\right]$ of $A$ (cf. [Se1, Définition 2.1]). We set

$$
B=\left\{x \in A \mid x z=z x \text { for all } z \in L_{\zeta}\right\} .
$$

Then we have $B=L_{\zeta}$. Using this, we see that the critical exponent of this stratum equals -1 (cf. [Se1, 2.1]). Hence, this stratum is simple (cf. [Se1, Définition 2.3]). Since the simple pair $\left[0, \varphi_{\zeta}^{-1}\right]$ over $K$ is minimal in the sense of [Se1, 2.3.3], we have

$$
H^{1}\left(\varphi_{\zeta}^{-1}, \mathfrak{A}\right)=J^{1}\left(\varphi_{\zeta}^{-1}, \mathfrak{A}\right)=U_{\mathfrak{A}}^{1} \subset J\left(\varphi_{\zeta}^{-1}, \mathfrak{A}\right)=\mathcal{O}_{L_{\zeta}}^{\times} U_{\mathfrak{A}}^{1}
$$

under the notation in [Se1, (65)]. We have

$$
\mathscr{C}\left(\varphi_{\zeta}^{-1}, 0, \mathfrak{A}\right)=\left\{\left.\theta_{D, m, \eta}\right|_{U_{\mathfrak{A}}^{1}}\right\}
$$

by [Se1, Lemma 3.23] under the notation in [Se1, Définition 3.45]. Then, by (1.2), (1.3) and (1.4), we can check that the pair

$$
(\mathrm{J}, \lambda)=\left(\mathcal{O}_{L_{\zeta}}^{\times} U_{\mathfrak{A}}^{1},\left.\theta_{D, m, \eta}\right|_{\mathcal{O}_{L_{\zeta}}^{\times} U_{\mathfrak{A}}^{1}}\right)
$$

is a maximal simple type of level $>0$ for $G$ with respect to the simple stratum $\left[\mathfrak{A}, 1,0, \varphi_{\zeta}^{-1}\right]$ in the sense of [Se3, 4.1 and 5.1]. Hence, $\pi_{D, m, \eta}$ is a supercuspidal representation by [Se3, Théorème 5.2]. We see $c\left(\pi_{D, m, \eta}\right)=n+1$ by using [BF, Theorem 3.3.8] (cf. the proof of [ABPS, Proposition 2.6]).

Proposition 1.3. The map

$$
\Phi: \mu_{q-1}(K) \times\left(k^{\times}\right)^{\vee} \times \mathbb{C}^{\times} \rightarrow \mathcal{A}_{D, m}^{n+1} ; \eta \mapsto \pi_{D, m, \eta}
$$

is a bijection.

Proof. We show the injectivity. We take

$$
\eta=(\zeta, \chi, c), \eta^{\prime}=\left(\zeta^{\prime}, \chi^{\prime}, c^{\prime}\right) \in \mu_{q-1}(K) \times\left(k^{\times}\right)^{\vee} \times \mathbb{C}^{\times}
$$

such that $\pi_{D, m, \eta} \simeq \pi_{D, m, \eta^{\prime}}$. By [SS2, Corollary 7.3], we see that $\zeta=\zeta^{\prime}$ and $\chi=\chi^{\prime}$. By $\pi_{D, m, \eta} \simeq \pi_{D, m, \eta^{\prime}}$, there exists $g_{0} \in G$ such that

$$
\operatorname{Hom}_{L_{\zeta}^{\times} U_{\mathfrak{A}}^{1} \cap\left(L_{\zeta}^{\times} U_{\mathfrak{A}}^{1}\right)^{g_{0}}}\left(\theta_{D, m, \eta}, \theta_{D, m, \eta^{\prime}}^{g_{0}}\right) \neq 0 .
$$

This implies

$$
\operatorname{Hom}_{U_{\mathfrak{A}}^{1} \cap\left(U_{\mathfrak{A}}^{1}\right)^{g_{0}}}\left(\theta_{D, m, \eta}, \theta_{D, m, \eta}^{g_{0}}\right) \neq 0,
$$


since $\theta_{D, m, \eta}$ coincides with $\theta_{D, m, \eta^{\prime}}$ on $U_{\mathfrak{A} \text {. }}^{1}$ Then we have $g_{0} \in L_{\zeta}^{\times} U_{\mathfrak{A}}^{1}$ by $[$ Se2, Proposition 2.10]. Hence, we have $\eta=\eta^{\prime}$ by (1.5).

We show the surjectivity. Let $\pi \in \mathcal{A}_{D, m}^{n+1}$. By [SS1, Théorème 5.21 and Corollaire 5.22 ], we have $\pi \simeq \mathrm{c}-\operatorname{Ind}_{\bar{J}}^{G} \theta$ for a maximal simple type $(J, \lambda)$ with a simple stratum $\left[\mathfrak{A}_{0}, l, 0, \beta\right]$ and an extension $\theta$ of $\lambda$ to

$$
\bar{J}=\left\{g \in G \mid J^{g}=J, \lambda^{g}=\lambda\right\} .
$$

Let $\mathfrak{P}_{\mathfrak{A}_{0}}$ be the Jacobson radical of $\mathfrak{A}_{0}$. By [ABPS, Proposition 2.6], we have $l=1$ and $\mathfrak{p}_{K} \mathfrak{A}_{0}=\mathfrak{P}_{\mathfrak{A}_{0}}^{n}$. Replacing $\mathfrak{A}_{0}$ by its conjugate, we may assume that $\mathfrak{A}_{0}=\mathfrak{A}$ (cf. [BF, (1.5.2) Proposition (ii)]). We put $U_{K}^{1}=1+\mathfrak{p}_{K}$. We take $\zeta \in \mu_{q-1}(K)$ such that

$$
\operatorname{Nrd}_{A / K}\left(\beta^{-1}\right) \equiv(-1)^{n-1} \zeta \varpi \bmod U_{K}^{1}
$$

We write $\beta^{-1}=\varphi_{\zeta} a u$, where $u \in U_{\mathfrak{A}}^{1}$ and $a=\left(a_{i j}\right)_{1 \leq i, j \leq m} \in \mathfrak{A}^{\times}$is a diagonal matrix such that $a_{i i} \in \mu_{q^{r}-1}\left(K_{r}\right)$ for $1 \leq i \leq m$. We put $b=\prod_{1 \leq i \leq m} a_{i i}$. Then we see that $\varphi_{\zeta} a$ is conjugate to

$$
\left(\begin{array}{cc}
\mathbf{0} & I_{m-1} \\
\varphi_{D, \zeta} b & \mathbf{0}
\end{array}\right) \in A
$$

by an element of $\mathfrak{A}^{\times}$. Further, this is conjugate to $\varphi_{\zeta}$ by an element of $\mathfrak{A}^{\times}$, since we have $\operatorname{Nrd}_{D / K}(b)=1$ by (1.6). Hence, we may assume that $\beta=\varphi_{\zeta}^{-1}$.

We see that $\theta$ is a character by the definition of a maximal simple type (cf. [Se3, 4.1 and 5.1]), [Se2, 2.2] and (1.3). We define $\chi \in\left(k^{\times}\right)^{\vee}$ by $\chi(\bar{x})=\theta(x)$ for $x \in \mu_{q-1}(K)$, and put $c=(-1)^{m-1} \theta\left(\varphi_{\zeta}\right)$. Then we have $\pi=\Phi(\eta)$ for $\eta=(\zeta, \chi, c)$.

Remark 1.4. If $m=n$, Proposition 1.3 follows from [BH6, 2.1 and 2.2]. Actually, the proof of the injectivity of $\Phi$ is logically unnecessary in this paper: We need only the surjectivity of $\Phi$ in the case $m=n$ for the proof of Theorem 3.5, and the injectivity of $\Phi$ follows from Theorem 3.5 and the injectivity of $\Phi$ in the case $m=n$.

\section{Formula for character}

In this section, we give formulas for characters of the simple supercuspidal representations of $G$ at some elements.

Definition 2.1 (cf. [BH5, 1.1 Remark]). Let $g \in G$. Let $f_{g}(x) \in K[x]$ be the reduced characteristic polynomial of $g$ over $K$.

1. We say that $g$ is regular if $f_{g}(x)$ is separable.

2. We say that $g$ is quasi-regular if $f_{g}(x)$ has no repeated irreducible factor over $K$.

3. We say that $g$ is elliptic if the minimal polynomial of $g$ is irreducible over $K$.

We write $G_{\mathrm{reg}}, G_{\mathrm{qr}}$ and $G_{\mathrm{qr}}^{\text {ell }}$ for the set of the regular elements of $G$, the set of the quasi-regular elements of $G$ and the set of the elliptic quasi-regular elements of $G$ respectively.

Let $\mathcal{H}(G)$ be the space of locally constant compactly supported functions $G \rightarrow \mathbb{C}$. We take a Haar measure $d \mu_{G}$ on $G$. We recall the following fact due to BushnellHenniart: 
Proposition 2.2. For an irreducible smooth representation $\pi$ of $G$, there is a locally constant function $\operatorname{tr} \pi: G_{\mathrm{qr}} \rightarrow \mathbb{C}$ characterized by

$$
\operatorname{tr} \pi(f)=\int_{G} \operatorname{tr} \pi(g) f(g) d \mu_{G}
$$

for all $f \in \mathcal{H}(G)$ with support contained in $G_{\mathrm{qr}}$.

Proof. This is proved in [BH1, (A.11) Corollary] in the case where $G=G L_{n}(K)$. The same arguments work also in our situation (cf. [BH5, 1.1 Remark and Proposition]).

For an irreducible smooth representation $\pi$ of $G$, let $\operatorname{tr} \pi$ be the function in Proposition 2.2, which we call the character of $\pi$.

Let $\eta=(\zeta, \chi, c) \in \mu_{q-1}(K) \times\left(k^{\times}\right)^{\vee} \times \mathbb{C}^{\times}$.

Lemma 2.3. We have

$$
\operatorname{tr} \pi_{D, m, \eta}(g)=\sum_{x \in G / L_{\zeta}^{\times} U_{\mathfrak{A}}^{1}, x^{-1} g x \in L_{\zeta}^{\times} U_{\mathfrak{A}}^{1}} \theta_{D, m, \eta}\left(x^{-1} g x\right)
$$

for $g \in G_{\mathrm{qr}}^{\mathrm{ell}}$.

Proof. This follows from [BH5, 1.1 Remark and (1.2.2)] (cf. [BH1, (A.14)]).

We put $U_{K}^{1}=1+\mathfrak{p}_{K} \subset \mathcal{O}_{K}^{\times}$. We set $g_{u}=\varphi_{\zeta}\left(1+\varphi_{\zeta} u\right) \in \mathfrak{A}$ for $u \in \mathfrak{A}$.

Lemma 2.4. Let $u \in \mathfrak{A}$. We set

$$
f_{g_{u}}(x)=x^{n}+\sum_{i=0}^{n-1} a_{i}(u) x^{i} \in K[x] .
$$

Then, we have

$$
a_{i}(u) \in \mathfrak{p}_{K} \quad \text { for } 1 \leq i \leq n-1, \quad-\frac{a_{0}(u)}{\zeta \varpi} \in U_{K}^{1} .
$$

Furthermore, $g_{u}$ is an elliptic quasi-regular element.

Proof. We see that $a_{i}(u) \in \mathfrak{p}_{K}$ for $0 \leq i \leq n-1$, since the left multiplication on $\mathcal{O}_{D} / \varpi \mathcal{O}_{D}$ by $g_{u}$ acts nilpotently. We have

$$
a_{0}(u)=(-1)^{n} \operatorname{Nrd}_{A / K}\left(g_{u}\right) \in \mathfrak{p}_{K} \backslash \mathfrak{p}_{K}^{2} .
$$

Hence, the former assertion follows from (1.1) and $\operatorname{Nrd}_{A / K}\left(1+\varphi_{\zeta} u\right) \in U_{K}^{1}$. By Eisenstein's irreducibility criterion, $f_{g_{u}}(x)$ is irreducible over $K$. Therefore, the latter assertion follows.

Lemma 2.5. Let $g \in G$ and $u \in \mathfrak{A}$. Assume that $g^{-1} g_{u} g \in \mathfrak{P}_{\mathfrak{A}}$. Then we have $g \in L_{\zeta}^{\times} \mathfrak{A}^{\times}$. 
Proof. We take $l \in \mathbb{Z}$ and $s=\left(s_{i, j}\right)_{1 \leq i, j \leq m} \in \mathfrak{A} \backslash \mathfrak{P}_{\mathfrak{A}}$ such that $g=\varphi_{\zeta}^{l} s$. We put $u^{\prime}=\varphi_{\zeta}^{-l} u \varphi_{\zeta}^{l} \in \mathfrak{A}$. Then we have

$$
g^{-1} g_{u} g=s^{-1} g_{u^{\prime}} s \in \mathfrak{P}_{\mathfrak{A}} .
$$

Hence, we have $g_{u^{\prime}} s \mathfrak{A} \subset s \varphi_{\zeta} \mathfrak{A}$. This implies $g_{u^{\prime}} s \mathfrak{A}=s \varphi_{\zeta} \mathfrak{A}$, since we have

$$
\operatorname{Nrd}_{A / K}\left(\varphi_{\zeta}^{-1} s^{-1} g_{u^{\prime}} s\right) \in \mathcal{O}_{K}^{\times}
$$

Therefore we have $\varphi_{\zeta} s \mathfrak{A}=s \varphi_{\zeta} \mathfrak{A}$ in $\mathfrak{P}_{\mathfrak{A}} / \mathfrak{P}_{\mathfrak{A}}^{2}$. This implies that $s_{i, i} \in \mathcal{O}_{D}^{\times}$if and only if $s_{i+1, i+1} \in \mathcal{O}_{D}^{\times}$for each $1 \leq i \leq m-1$. Then we have $s_{i, i} \in \mathcal{O}_{D}^{\times}$for all $1 \leq i \leq m$, since $s \notin \mathfrak{P}_{\mathfrak{A}}$. Hence, we have $s \in \mathfrak{A}^{\times}$. This shows the claim.

We put $n_{q}=(n, q-1)$. For $a \in k$, we put

$$
G_{n}(\chi, \psi, a)=\sum_{x \in \mu_{n_{q}}(k)} \chi(x) \psi(a x)
$$

Note that $G_{q-1}(\chi, \psi, 1)$ is a usual Gauss sum, for which we write $G(\chi, \psi)$.

Proposition 2.6. For $u \in \mathfrak{A}$, we have

$$
\operatorname{tr} \pi_{D, m, \eta}\left(g_{u}\right)=(-1)^{m-1} c G_{n}\left(\chi, \psi, \overline{\operatorname{Trd}_{A / K}(u)}\right) .
$$

Proof. For $\lambda \in \mu_{q-1}(K)$, let

$$
g(\lambda)=\operatorname{diag}\left(1, \lambda, \cdots, \lambda^{m-1}\right) \in G .
$$

We put

$$
H=\left\{d g(\lambda) \in G \mid d \in \mu_{q^{r}-1}\left(K_{r}\right), \lambda \in \mu_{q-1}(K), d^{q^{s}-1}=\lambda^{m}\right\} .
$$

Note that $\lambda$ in the definition of $H$ automatically belongs to $\mu_{n_{q}}(K)$. Then, we can check that $H$ is equal to

$$
\left\{x=\operatorname{diag}\left(d_{1}, \cdots, d_{m}\right) \in G \mid x^{-1} g_{u} x \in L_{\zeta}^{\times} U_{\mathfrak{A}}^{1}, d_{i} \in \mu_{q^{r}-1}\left(K_{r}\right) \text { for all } i\right\} .
$$

Hence, we see that

$$
\left\{x \in G \mid x^{-1} g_{u} x \in L_{\zeta}^{\times} U_{\mathfrak{A}}^{1}\right\}=H L_{\zeta}^{\times} U_{\mathfrak{A}}^{1}
$$

by Lemma 2.5. We have the isomorphism

$$
H L_{\zeta}^{\times} U_{\mathfrak{A}}^{1} / L_{\zeta}^{\times} U_{\mathfrak{A}}^{1} \stackrel{\sim}{\rightarrow} \mu_{n_{q}}(k) ; d g(\lambda) \mapsto \bar{\lambda} .
$$

Hence, the claim follows from Lemma 2.3 and Lemma 2.4.

Lemma 2.7. The element $1+\varphi_{\zeta}$ is elliptic quasi-regular.

Proof. The element $\varphi_{\zeta}$ is elliptic quasi-regular by Lemma 2.4. Hence, the claim follows.

Lemma 2.8. Let $g \in G$ and $\lambda \in \mu_{q-1}(K)$. Assume that $g^{-1}\left(1+\varphi_{\zeta \lambda}\right) g \in L_{\zeta}^{\times} U_{\mathfrak{A}}^{1}$. Then we have $g \in L_{\zeta}^{\times} \mathfrak{A}^{\times}$. 
Proof. By the assumption, we have $g^{-1}\left(1+\varphi_{\zeta \lambda}\right) g=\lambda_{0}(1+v)$ with $\lambda_{0} \in \mu_{q-1}(K)$ and $v \in \mathfrak{P}_{\mathfrak{A}}$. Then we have

$$
\lambda_{0}=\lim _{l \rightarrow \infty}\left(\lambda_{0}(1+v)\right)^{q^{l}}=\lim _{l \rightarrow \infty}\left(g^{-1}\left(1+\varphi_{\zeta \lambda}\right) g\right)^{q^{l}}=1 .
$$

Hence we have $g^{-1} \varphi_{\zeta \lambda} g=v \in \mathfrak{P}_{\mathfrak{A}}$. Therefore, the claim follows from Lemma 2.5 and $L_{\zeta}^{\times} \mathfrak{A}^{\times}=L_{\zeta \lambda}^{\times} \mathfrak{A}^{\times}$.

For a finite field extension $k^{\prime}$ of $k$, an additive character $\psi^{\prime} \in k^{\prime \vee}$, a positive integer $l$ and $a \in k^{\prime \times}$, we put

$$
K_{l, a}\left(\psi^{\prime}\right)=\sum_{\zeta_{1} \cdots \zeta_{l}=a, \zeta_{i} \in k^{\prime} \times} \psi^{\prime}\left(\sum_{i=1}^{l} \zeta_{i}\right) .
$$

This is a generalized Kloosterman sum (cf. [De, Sommes trig. 7.1]).

Proposition 2.9. For $\lambda \in \mu_{q-1}(K)$, we have

$$
\operatorname{tr} \pi_{D, m, \eta}\left(1+\varphi_{\zeta \lambda}\right)=(-1)^{n-m} K_{n, \bar{\lambda}}(\psi) .
$$

Proof. Let $\lambda \in \mu_{q-1}(K)$. We have

$$
\operatorname{tr} \pi_{D, m, \eta}\left(1+\varphi_{\zeta \lambda}\right)=\sum_{g \in L_{\zeta}^{\times} \mathfrak{A} \times / L_{\zeta}^{\times} U_{\mathfrak{A}}^{1}} \theta_{D, m, \eta}\left(g^{-1}\left(1+\varphi_{\zeta \lambda}\right) g\right)
$$

by Lemma 2.3, Lemma 2.7 and Lemma 2.8, We can check that

$$
\operatorname{tr}_{A / D}\left(\varphi_{\zeta}^{-1} g^{-1} \varphi_{\zeta \lambda} g\right) \equiv \varphi_{D, \zeta}^{-1} a_{m, m}^{-1} \varphi_{D, \zeta \lambda} a_{1,1}+\sum_{i=1}^{m-1} a_{i, i}^{-1} a_{i+1, i+1} \quad \bmod \mathfrak{p}_{D}
$$

for $g=\left(a_{i, j}\right)_{1 \leq i, j \leq m} \in \mathfrak{A}^{\times}$. For $d \in \mathcal{O}_{D}$, let $\bar{d}$ denote the image of $d$ by $\mathcal{O}_{D} \rightarrow k_{r}$. We have the bijection

$$
\begin{aligned}
L_{\zeta}^{\times} \mathfrak{A}^{\times} / L_{\zeta}^{\times} U_{\mathfrak{A}}^{1} \stackrel{\sim}{\rightarrow}\left\{\left(\zeta_{1}, \cdots, \zeta_{m}\right) \in\left(k_{r}^{\times}\right)^{\oplus m} \mid \operatorname{Nr}_{k_{r} / k}\left(\zeta_{1} \cdots \zeta_{m}\right)=\bar{\lambda}\right\} \\
\left(a_{i, j}\right)_{1 \leq i, j \leq m} \mapsto\left(\left(\bar{a}_{i, i}^{-1} \bar{a}_{i+1, i+1}\right)_{1 \leq i \leq m-1}, \overline{\varphi_{D, \zeta}^{-1} a_{m, m}^{-1} \varphi_{D, \zeta \lambda} a_{1,1}}\right),
\end{aligned}
$$

where $\left(a_{i, j}\right)_{1 \leq i, j \leq m} \in \mathfrak{A}^{\times}$. Hence, we have

$$
\operatorname{tr} \pi_{D, m, \eta}\left(1+\varphi_{\zeta \lambda}\right)=\sum_{\mathrm{Nr}_{k_{r} / k}(x)=\bar{\lambda}} K_{m, x}\left(\psi \circ \operatorname{Tr}_{k_{r} / k}\right)
$$

Further, we have

$$
\sum_{\mathrm{Nr}_{k_{r} / k}(x)=\bar{\lambda}} K_{m, x}\left(\psi \circ \operatorname{Tr}_{k_{r} / k}\right)=(-1)^{m-1} \sum_{\operatorname{Nr}_{k_{n} / k}(y)=\bar{\lambda}} \psi \circ \operatorname{Tr}_{k_{n} / k}(y)=(-1)^{n-m} K_{n, \bar{\lambda}}(\psi)
$$

by [De, (7.2.5)]. Thus, we have proved the claim. 


\section{Description of LJLC}

In this section, we give an explicit description of the local Jacquet-Langlands correspondence (LJLC).

First, we recall the statement of the LJLC. Let $\mathcal{A}_{D, m}^{\square}$ denote the set of equivalent classes of irreducible essentially square-integrable smooth representations of $G L_{m}(D)$. We put $G^{\prime}=G L_{n}(K)$. We say that $g \in G_{\mathrm{qr}}$ and $g^{\prime} \in G_{\mathrm{qr}}^{\prime}$ correspond if $f_{g}(x)=f_{g^{\prime}}(x)$.

Theorem 3.1 ([Ba, Théorème 5.1] and [DKV, Théorème principal]). There exists a bijection

$$
\mathrm{JL}_{D, m}: \mathcal{A}_{D, m}^{\square} \rightarrow \mathcal{A}_{K, n}^{\square},
$$

which is uniquely characterized by the character relation

$$
\operatorname{tr} \pi(g)=(-1)^{n-m} \operatorname{tr} \mathrm{JL}_{D, m}(\pi)\left(g^{\prime}\right)
$$

for $\pi \in \mathcal{A}_{D, m}^{\square}$ and any corresponding $g \in G_{\mathrm{reg}}$ and $g^{\prime} \in G_{\mathrm{reg}}^{\prime}$.

The bijection $\mathrm{JL}_{D, m}$ in Theorem 3.1 is called the local Jacquet-Langlands correspondence.

Corollary 3.2. The bijection $\mathrm{JL}_{D, m}$ satisfies the character relation

$$
\operatorname{tr} \pi(g)=(-1)^{n-m} \operatorname{tr} \mathrm{JL}_{D, m}(\pi)\left(g^{\prime}\right)
$$

for $\pi \in \mathcal{A}_{D, m}^{\square}$ and any corresponding $g \in G_{\mathrm{qr}}$ and $g^{\prime} \in G_{\mathrm{qr}}^{\prime}$.

Proof. The complement of $G^{\text {reg }}$ in $G$ is a Zariski closed subset of $G$ with strictly smaller dimension than $G$. This implies that $G^{\text {reg }}$ is dense in $G$ in the $\varpi$-adic topology, hence it is dense also in $G^{\mathrm{qr}}$. Therefore, the claim follows from Theorem 3.1, since $\operatorname{tr} \pi$ and $\operatorname{tr} \mathrm{JL}_{D, m}(\pi)$ for $\pi \in \mathcal{A}_{D, m}^{\square}$ are locally constant functions on $G_{\mathrm{qr}}$ and $G_{\mathrm{qr}}^{\prime}$ respectively.

Lemma 3.3. The function

$$
G_{n, \chi, \psi}: k \rightarrow \mathbb{C} ; a \mapsto G_{n}(\chi, \psi, a) .
$$

is not equal to the zero function on $k$.

Proof. Let $F_{n, \chi}$ be the function on $k$ defined by

$$
F_{n, \chi}(x)= \begin{cases}\chi(x) & \text { if } x \in \mu_{n_{q}}(k), \\ 0 & \text { if } x \notin \mu_{n_{q}}(k) .\end{cases}
$$

Then $G_{n, \chi, \psi}$ is regarded as the Fourier transformation of $F_{n, \chi}$ with respect to $\psi$. Hence, the claim follows from the Fourier inversion formula.

Lemma 3.4. For any $a^{\prime} \in k^{\times} \backslash\{1\}$, there is $a \in k^{\times}$such that $K_{n, a}(\psi) \neq K_{n, a a^{\prime}}(\psi)$.

Proof. Let $a^{\prime} \in k^{\times} \backslash\{1\}$. We take $\chi^{\prime} \in\left(k^{\times}\right)^{\vee}$ such that $\chi^{\prime}\left(a^{\prime}\right) \neq 1$. Then we have

$$
\begin{aligned}
\sum_{a \in k^{\times}} \chi^{\prime}\left(a a^{\prime}\right)\left(K_{n, a}(\psi)-K_{n, a a^{\prime}}(\psi)\right) & =\left(\chi^{\prime}\left(a^{\prime}\right)-1\right) \sum_{a \in k^{\times}} \chi^{\prime}(a) K_{n, a}(\psi) \\
& =\left(\chi^{\prime}\left(a^{\prime}\right)-1\right) G\left(\chi^{\prime}, \psi\right)^{n} \neq 0
\end{aligned}
$$

by [De, (7.1.6)]. Hence, the claim follows. 
Theorem 3.5. For $\eta=(\zeta, \chi, c) \in \mu_{q-1}(K) \times\left(k^{\times}\right)^{\vee} \times \mathbb{C}^{\times}$, we have $\mathrm{JL}_{D, m}\left(\pi_{D, m, \eta}\right)=$ $\pi_{K, n, \eta} \cdot$

Proof. Let $\mathrm{LJ}_{D, m}$ denote the inverse of $\mathrm{JL}_{D, m}$. The map $\mathrm{LJ}_{D, m}$ preserves conductors and supercuspidality by ABPS, Theorem 2.1(e), (i)]. Let $\eta^{\prime}=\left(\zeta^{\prime}, \chi^{\prime}, c^{\prime}\right) \in \mu_{q-1}(K) \times$ $\left(k^{\times}\right)^{\vee} \times \mathbb{C}^{\times}$. By Proposition 1.3, there exists $\eta=(\zeta, \chi, c) \in \mu_{q-1}(K) \times\left(k^{\times}\right)^{\vee} \times \mathbb{C}^{\times}$such that $\mathrm{LJ}_{D, m}\left(\pi_{K, n, \eta^{\prime}}\right)=\pi_{D, m, \eta}$.

We have to show $\eta=\eta^{\prime}$. We have $\chi=\chi^{\prime}$, because $\mathrm{JL}_{D, m}$ preserves central characters. We write $\mathfrak{A}^{\prime}$ and $\varphi_{\zeta}^{\prime}$ for $\mathfrak{A}$ and $\varphi_{\zeta}$ respectively in the case $m=n$. By Proposition 2.9 and Corollary [3.2, we have

$$
K_{n, \bar{\lambda}}(\psi)=(-1)^{n-m} \operatorname{tr} \pi_{D, m, \eta}\left(1+\varphi_{\zeta \lambda}\right)=\operatorname{tr} \pi_{K, n, \eta^{\prime}}\left(1+\varphi_{\zeta \lambda}^{\prime}\right)=K_{n, \overline{\lambda \zeta \zeta^{\prime-1}}}(\psi)
$$

for $\lambda \in \mu_{q-1}(K)$. Hence, we have $\zeta=\zeta^{\prime}$ by Lemma 3.4 .

Finally, we show $c=c^{\prime}$. By Lemma 3.3, we can take $a \in k$ such that $G_{n}(\chi, \psi, a) \neq 0$. We take $\xi_{0} \in k_{r}$ such that $\operatorname{Tr}_{k_{r} / k}\left(\xi_{0}\right)=a$. Let $\xi \in \mathcal{O}_{D}$ be a lifting of $\xi_{0}$. We set

$$
u_{\xi}=\operatorname{diag}(\xi, 0, \cdots, 0) \in \mathfrak{A} .
$$

We simply write $g_{\xi}$ for $g_{u_{\xi}}$. Let $\left\{a_{i}\left(u_{\xi}\right)\right\}_{0 \leq i \leq n-1}$ be as in Lemma 2.4. By Lemma 2.4. the elements

$$
\alpha_{i}=-\frac{a_{i}\left(u_{\xi}\right)}{\zeta \varpi} \quad \text { for } 1 \leq i \leq n-1, \quad \alpha_{n}=-\frac{\frac{a_{0}\left(u_{\xi}\right)}{\zeta \varpi}+1}{\zeta \varpi}
$$

are contained in $\mathcal{O}_{K}$. We set

$$
u_{\alpha}=\sum_{i=1}^{n} \varphi_{\zeta}^{\prime i-1} \operatorname{diag}\left(\alpha_{i}, 0, \cdots, 0\right) \in \mathfrak{A}^{\prime}, \quad g_{\alpha}=\varphi_{\zeta}^{\prime}\left(1+\varphi_{\zeta}^{\prime} u_{\alpha}\right) \in G^{\prime} .
$$

Then we have

$$
f_{g_{\alpha}}(x)=x^{n}-\sum_{i=1}^{n-1} \zeta \varpi \alpha_{i} x^{i}-\zeta \varpi\left(1+\alpha_{n} \zeta \varpi\right)=f_{g_{\xi}}(x) .
$$

Hence, $g_{\xi} \in G_{\mathrm{qr}}$ and $g_{\alpha} \in G_{\mathrm{qr}}^{\prime}$ correspond. We have $\overline{\operatorname{tr}\left(u_{\alpha}\right)}=\overline{\operatorname{Trd}_{A / K}\left(u_{\xi}\right)}=a$, since

$$
\operatorname{tr}\left(u_{\alpha}\right) \equiv \alpha_{1}=(-1)^{n} \frac{\operatorname{Trd}_{A / K}\left(g_{\xi}^{-1}\right) \operatorname{Nrd}_{A / K}\left(g_{\xi}\right)}{\zeta \varpi} \equiv \operatorname{Trd}_{D / K}(\xi) \quad \bmod \mathfrak{p}_{K} .
$$

Therefore, we have

$$
c=(-1)^{m-1} \frac{\operatorname{tr} \pi_{D, m, \eta}\left(g_{\xi}\right)}{G_{n}(\chi, \psi, a)}=(-1)^{n-1} \frac{\operatorname{tr} \pi_{K, n, \eta^{\prime}}\left(g_{\alpha}\right)}{G_{n}(\chi, \psi, a)}=c^{\prime}
$$

by Proposition 2.6 and Corollary 3.2. 


\section{Another proof of Theorem 3.5}

Let $\eta=(\zeta, \chi, c) \in \mu_{q-1}(K) \times\left(k^{\times}\right)^{\vee} \times \mathbb{C}^{\times}$. We simply write $\epsilon\left(\pi_{D, m, \eta}, \psi_{K}\right)$ for the value of $\epsilon\left(\pi_{D, m, \eta}, s, \psi_{K}\right)$ at $1 / 2$. For any smooth character $\xi: K^{\times} \rightarrow \mathbb{C}^{\times}$, let $\xi_{A}$ denote the composite $\xi \circ \operatorname{Nrd}_{A / K}$.

The following lemma is a special case of a formula which represents GodementJacquet local constants with respect to non-abelian congruence Gauss sums. Such a formula is studied in $[\mathrm{Bu}]$ and $[\mathrm{BF}$. If $m=n$, this lemma is just [BH6, 2.2 Lemma].

Lemma 4.1. 1. We have

$$
\epsilon\left(\pi_{D, m, \eta}, \psi_{K}\right)=(-1)^{n-1} c .
$$

2. For any tamely ramified character $\xi$ of $K^{\times}$, we have

$$
\epsilon\left(\pi_{D, m, \eta} \otimes \xi_{A}, \psi_{K}\right)=\xi\left((-1)^{n-1} \zeta \varpi\right) \epsilon\left(\pi_{D, m, \eta}, \psi_{K}\right) .
$$

Proof. Let $\xi$ be a tamely ramified character of $K^{\times}$. Assume that the characteristic of $K$ equals zero. We set

$$
\rho=\operatorname{Ind}_{L_{\zeta}^{\times} U_{\mathfrak{A}}^{1}}^{L_{\widehat{A}}^{\times} \times}\left(\theta_{D, m, \eta} \otimes \xi_{A}\right) .
$$

We see that $\rho$ is an irreducible admissible representation of $L_{\zeta}^{\times} \mathfrak{A}^{\times}$by Lemma 1.2, and non-degenerate in the sense of [BF, p. 228] by (1.2).

Let $\psi_{K}^{0}$ be the standard continuous additive character of $K$ defined in [BF, 2.1]. In $[\mathrm{BF},(2.3 .6)]$, the non-abelian congruence Gauss sum $\tau(\rho)$ is defined for $\rho$ and $\psi_{K}^{0}$. However, it can be defined for any non-trivial additive character $\psi_{K}^{\prime}$ of $K$ as in $\underline{\mathrm{Bu}}$, (3.4)] (cf. [Bu, Remarks after Theorem (3.7)]), for which we write $\tau\left(\rho, \psi_{K}^{\prime}\right)$.

We take an element $a \in K^{\times}$such that $\psi_{K}(x)=\psi_{K}^{0}(a x)$ for $x \in K$. Let $\omega_{\rho} \vee$ denote the central character of $\rho^{\vee}$. By [Bu, (3.4)] and [GJ, (3.3.5)], we have formulas

$$
\begin{aligned}
\tau\left(\rho^{\vee}, \psi_{K}\right) & =\omega_{\rho^{\vee}}\left(a^{-1}\right) \tau\left(\rho^{\vee}, \psi_{K}^{0}\right)=\left(\theta_{D, m, \eta} \otimes \xi_{A}\right)(a) \tau\left(\rho^{\vee}, \psi_{K}^{0}\right), \\
\epsilon\left(\pi_{D, m, \eta} \otimes \xi_{A}, \psi_{K}\right) & =\left(\theta_{D, m, \eta} \otimes \xi_{A}\right)(a) \epsilon\left(\pi_{D, m, \eta} \otimes \xi_{A}, \psi_{K}^{0}\right)
\end{aligned}
$$

respectively. By these formulas and $[\mathrm{BF}$, Theorem (3.3.8)(iv)], we obtain

$$
\epsilon\left(\pi_{D, m, \eta} \otimes \xi_{A}, \psi_{K}\right)=(-1)^{n-m} \tau\left(\rho^{\vee}, \psi_{K}\right) \mathrm{Nf}(\rho)^{-1 / 2}
$$

We put $\psi_{A}=\psi_{K} \circ \operatorname{Trd}_{A / K}$. Then we have

$$
\tau\left(\rho^{\vee}, \psi_{K}\right)=\mathrm{Nf}(\rho)^{1 / 2}\left(\theta_{D, m, \eta} \otimes \xi_{A}\right)^{\vee}\left(\varphi_{\zeta}^{-1}\right) \psi_{A}\left(\varphi_{\zeta}^{-1}\right)
$$

by (4.1) and $[\mathrm{BF},(2.7 .4)]$. We obtain

$$
\begin{aligned}
\epsilon\left(\pi_{D, m, \eta} \otimes \xi_{A}, \psi_{K}\right) & =(-1)^{n-m}\left(\theta_{D, m, \eta} \otimes \xi_{A}\right)^{\vee}\left(\varphi_{\zeta}^{-1}\right) \psi_{A}\left(\varphi_{\zeta}^{-1}\right) \\
& =(-1)^{n-1} \xi\left((-1)^{n-1} \zeta \varpi\right) c
\end{aligned}
$$

by (1.1), (1.2), (4.2) and (4.3). The same arguments work also in the equal characteristic case (cf. [Bu, Remarks after Theorem (3.7)]). Hence, we obtain the claims. 
Another proof of Theorem 3.5. Let $\eta^{\prime}=\left(\zeta^{\prime}, \chi^{\prime}, c^{\prime}\right) \in \mu_{q-1}(K) \times\left(k^{\times}\right)^{\vee} \times \mathbb{C}^{\times}$. As in the first proof of Theorem 3.5, we see that there exists a triple $\eta=\left(\zeta, \chi^{\prime}, c\right) \in \mu_{q-1}(K) \times$ $\left(k^{\times}\right)^{\vee} \times \mathbb{C}^{\times}$such that $\pi_{D, m, \eta} \simeq \mathrm{LJ}_{D, m}\left(\pi_{K, n, \eta^{\prime}}\right)$. We prove $\zeta=\zeta^{\prime}$. Let $\xi$ be any tamely ramified character of $K^{\times}$. We write $A^{\prime}$ for $A$ in the case $m=n$. Since the $\mathrm{JL}_{D, m}$ preserves character twists, we have

$$
\epsilon\left(\pi_{D, m, \eta} \otimes \xi_{A}, \psi_{K}\right)=\epsilon\left(\pi_{K, n, \eta^{\prime}} \otimes \xi_{A^{\prime}}, \psi_{K}\right)
$$

by $\left[\right.$ ABPS, Theorem 2.2(i)] (cf. [DKV, B.j.1]). We have $\epsilon\left(\pi_{K, n, \eta^{\prime}}, \psi_{K}\right) \neq 0$ by Lemma 4.1.1. By Lemma 4.1,2 and (4.4), we have

$$
\xi\left((-1)^{n-1} \zeta \varpi\right)=\frac{\epsilon\left(\pi_{D, m, \eta} \otimes \xi_{A}, \psi_{K}\right)}{\epsilon\left(\pi_{D, m, \eta}, \psi_{K}\right)}=\frac{\epsilon\left(\pi_{K, n, \eta^{\prime}} \otimes \xi_{A^{\prime}}, \psi_{K}\right)}{\epsilon\left(\pi_{K, n, \eta^{\prime}}, \psi_{K}\right)}=\xi\left((-1)^{n-1} \zeta^{\prime} \varpi\right) .
$$

Hence, we obtain $\xi\left(\zeta \zeta^{\prime-1}\right)=1$. This implies $\zeta=\zeta^{\prime}$. By Lemma 4.1.1 and (4.4), we have

$$
c=(-1)^{n-1} \epsilon\left(\pi_{D, m, \eta}, \psi_{K}\right)=(-1)^{n-1} \epsilon\left(\pi_{K, n, \eta^{\prime}}, \psi_{K}\right)=c^{\prime} .
$$

Therefore we have $\eta=\eta^{\prime}$.

\section{$5 \quad$ Invariance of endo-class}

We show that endo-classes for the simple supercuspidal representations are invariant under the LJLC.

Let $\mathcal{E}(K)$ be the set of endo-classes of ps-characters over $K$ in the sense of BBSS, Definition 1.10]. Then we have a map

$$
\Theta_{G}: \mathcal{A}_{D, m}^{\square} \rightarrow \mathcal{E}(K)
$$

constructed in [BSS, 9.3]. Broussous-Sécherre-Stevens conjecture that

$$
\Theta_{G}(\pi)=\Theta_{G^{\prime}}\left(\mathrm{JL}_{D, m}(\pi)\right)
$$

for $\pi \in \mathcal{A}_{D, m}^{\square}$ in [BSS, Conjecture 9.5]. The following proposition verifies the conjecture for the simple supercuspidal representations.

Proposition 5.1. For any simple supercuspidal representation $\pi$ of $G$, we have

$$
\Theta_{G}(\pi)=\Theta_{G^{\prime}}\left(\mathrm{JL}_{D, m}(\pi)\right) .
$$

Proof. Let $\pi$ be a simple supercuspidal representation of $G$. We take $\eta=(\zeta, \chi, c) \in$ $\mu_{q-1}(K) \times\left(k^{\times}\right)^{\vee} \times \mathbb{C}^{\times}$such that $\pi=\pi_{D, m, \eta}$. Then $\Theta_{G}\left(\pi_{D, m, \eta}\right)$ is the endo-class of the ps-character defined by

$$
\left(\left[\mathfrak{A}, 1,0, \varphi_{\zeta}^{-1}\right],\left.\theta_{D, m, \eta}\right|_{U_{\mathfrak{A}}^{1}}\right) .
$$

We have $\mathrm{JL}_{D, m}\left(\pi_{D, m, \eta}\right)=\pi_{K, n, \eta}$ by Theorem 3.5. We define $\mathfrak{A}^{\prime}$ and $\varphi_{\zeta}^{\prime}$ for $M_{n}(K)$ similarly as $\mathfrak{A}$ and $\varphi_{\zeta}$ for $M_{m}(D)$. Then $\Theta_{G}\left(\pi_{K, n, \eta}\right)$ is the endo-class of the ps-character defined by

$$
\left(\left[\mathfrak{A}^{\prime}, 1,0, \varphi_{\zeta}^{\prime-1}\right],\left.\theta_{K, n, \eta}\right|_{U_{\mathfrak{A}^{\prime}}^{1}}\right) .
$$


Let $L_{\zeta, 0}$ be a finite extension of $K$ generated by an element $\varphi_{\zeta, 0}$ such that $\varphi_{\zeta, 0}^{n}=\zeta \varpi$. Let $\mathscr{C}_{K}\left(\varphi_{\zeta, 0}^{-1}, 0\right)$ be the set of simple characters corresponding to the simple pair $\left[\varphi_{\zeta, 0}^{-1}, 0\right]$ over $K$ defined in [Se1, p. 371]. Then we have bijective transfer maps

$$
\begin{aligned}
& \tau_{\mathfrak{A}, 0, \varphi_{\zeta, 0}^{-1}}: \mathscr{C}_{K}\left(\varphi_{\zeta, 0}^{-1}, 0\right) \rightarrow \mathscr{C}\left(\varphi_{\zeta}^{-1}, 0, \mathfrak{A}\right), \\
& \tau_{\mathfrak{A}^{\prime}, 0, \varphi_{\zeta, 0}^{-1}}: \mathscr{C}_{K}\left(\varphi_{\zeta, 0}^{-1}, 0\right) \rightarrow \mathscr{C}\left(\varphi_{\zeta}^{\prime-1}, 0, \mathfrak{A}^{\prime}\right) .
\end{aligned}
$$

by [Se1, Théorème 3.53]. We have

$$
\tau_{\mathfrak{A}, 0, \varphi_{\zeta, 0}^{-1}}^{-1}\left(\left.\theta_{D, m, \eta}\right|_{U_{\mathfrak{A}}^{1}}\right)=\tau_{\mathfrak{A}^{\prime}, 0, \varphi_{\zeta, 0}^{-1}}^{-1}\left(\left.\theta_{K, n, \eta}\right|_{U_{\mathfrak{\mathfrak { I } ^ { \prime }}}^{1}}\right)
$$

by (1.4). Hence, we have the claim.

\section{References}

[AL] M. Adrian and B. Liu, Some results on simple supercuspidal representations of GL(n,F), J. Number Theory 160 (2016), 117-147.

[ABPS] A. M. Aubert, P. Baum, R. Plymen and M. Solleveld, Depth and the local Langlands correspondence, to appear in the memorial volume for F. Hirzebruch. arXiv:1311.1606v2.

[Ba] A. I. Badulescu, Correspondance de Jacquet-Langlands pour les corps locaux de caractéristique non nulle, Ann. Sci. École Norm. Sup. (4) 35 (2002), no. 5, 695-747.

[BSS] P. Broussous, V. Sécherre and S. Stevens, Smooth representations of $\operatorname{GL}_{m}(D)$ V: Endo-classes, Doc. Math. 17 (2012), 23-77.

[Bu] C. J. Bushnell, Gauss sums and local constants for $\mathrm{GL}(N)$, in L-functions and Arithmetic, London Mathematical Society Lecture Note Series 153, Cambridge university press, 1991, 61-75.

[BF] C. J. Bushnell and and A. Fröhlich, Non-abelian congruence Gauss sums and p-adic simple algebras, Proc. London Math. Soc. (3) 50 No. 2 (1985), 207-264.

[BH1] C. J. Bushnell and G. Henniart, Local tame lifting for GL(N) I: Simple characters, Inst. Hautes Études Sci. Publ. Math. No. 83 (1996), 105-233.

[BH2] C. J. Bushnell and G. Henniart, Correspondance de Jacquet-Langlands explicite II. Le cas de degré égal à la caractéristique résiduelle, Manuscripta Math. 102 (2000), no. 2, 211-225.

[BH3] C. J. Bushnell and G. Henniart, Local tame lifting for GL $(n)$ III: explicit base change and Jacquet-Langlands correspondence, J. reine angew. Math. 580 (2005), 39-100.

[BH4] C. J. Bushnell and G. Henniart, The local Langlands conjecture for GL(2), Grundlehren der Mathematischen Wissenschaften 335, Springer-Verlag, Berlin, 2006. 
[BH5] C. J. Bushnell and G. Henniart, The essentially tame Jacquet-Langlands correspondence for inner forms of GL( $n)$, Pure Appl. Math. Q. 7 (2011), no. 3, Special Issue: In honor of Jacques Tits, 469-538.

[BH6] C. J. Bushnell and G. Henniart, Langlands parameters for epipelagic representaions of $\mathrm{GL}_{n}$, Math. Ann. 358 (2014), no. 1-2, 433-463.

[BK] C. J. Bushnell and P.C. Kutzko, The admissible dual of GL(N) via compact open subgroups, Annals of Mathematics Studies, 129, Princeton University Press, Princeton, NJ, 1993.

[De] P. Deligne, Cohomologie étale, Lecture Notes in Mathematics, vol. 569, Springer-Verlag, Berlin-New York, 1977.

[DKV] P. Deligne, D. Kazhdan, M.-F. Vigneras, Représentations des algèbres centrales simples p-adiques, in Représentations des groupes réductifs sur un corps local, Travaux en cours, Hermann (1984), 33-117.

[Ge] P. Gérardin, Facteurs locaux des algèbres simples de rang 4. I, Reductive groups and automorphic forms, I (Paris, 1976/1977), pp. 37-77, Publ. Math. Univ. Paris VII, 1, Univ. Paris VII, Paris, 1978.

[GJ] R. Godement and H. Jacquet, Zeta functions of simple algebras, Lecture Notes in Mathematics 260, Springer-Verlag, Berlin-New York, 1970.

[He] G. Henniart, Correspondance de Jacquet-Langlands explicite. I. Le cas modéré de degré premier, Séminaire de Théorie des Nombres, Paris, 1990-91, 85-114, Progr. Math., 108, Birkhäuser Boston, Boston, MA, 1993.

[IT1] N. Imai and T. Tsushima, Affinoids in the Lubin-Tate perfectoid space and simple supercuspidal representations I: tame case, preprint. arXiv:1308.1276.

[IT2] N. Imai and T. Tsushima, Affinoids in the Lubin-Tate perfectoid space and simple supercuspidal representations II: wild case, preprint. arXiv:1603.04693.

[Ka] K. Kariyama, Endo-class and the Jacquet-Langlands correspondence, Kyoto. J. Math. 55 (2015), No. 2, 299-320.

[KL] A. Knightly and C. Li, Simple supercuspidal representations of GL( $n)$, Taiwanese J. Math. 19 (2015), no. 4, 995-1029.

[RY] M. Reeder and J.-K. Yu, Epipelagic representations and invariant theory, J. Amer. Math. Soc. 27 (2014), no. 2, 437-477.

[Se1] V. Sécherre, Représentations lisses de GL $(m, D)$ I: Caractères simples, Bull. Soc. Math. France 132 (2004), no. 3, 327-396.

[Se2] V. Sécherre, Représentations lisses de GL $(m, D)$ II: $\beta$-extensions, Compositio. Math. 141 (2005), no. 3, 1531-1550.

[Se3] V. Sécherre, Représensations lisses de $\mathrm{GL}_{m}(D)$ III: Types simples, Ann. Scient. Éc. Norm. Sup. 38 (2005), 951-977. 
[SS1] V. Sécherre and S. Stevens, Représentations lisses de $\mathrm{GL}_{m}(D)$ IV: Représentations supercuspidales, J. Inst. Math. Jussieu 7 (2008), no. 3, 527574 .

[SS2] V. Sécherre and S. Stevens, Smooth representations of $\mathrm{GL}_{m}(D)$ VI: semisimple types, Int. Math. Res. Not. IMRN 2012, no. 13, 2994-3039.

[SZ] A. J. Silberger and E.-W. Zink, An explicit matching theorem for level zero discrete series of unit groups of $\mathfrak{p}$-adic simple algebras, J. reine angew. Math. 585 (2005), 173-235.

[Xu] P. Xu, A remark on the simple cuspidal representations of $\mathrm{GL}(n, F)$, preprint. arXiv:1310.3519.

Naoki Imai

Graduate School of Mathematical Sciences, The University of Tokyo, 3-8-1 Komaba, Meguro-ku, Tokyo, 153-8914, Japan

naoki@ms.u-tokyo.ac.jp

Takahiro Tsushima

Department of Mathematics and Informatics, Faculty of Science, Chiba University, 1-33 Yayoi-cho, Inage, Chiba, 263-8522, Japan

tsushima@math.s.chiba-u.ac.jp 